\title{
Optic nerve head changes in early glaucoma: a comparison between stereophotography and Heidelberg retina tomography
}

LE Pablo', A Ferreras', P Fogagnolo², M Figus ${ }^{3}$ and AB Pajarin ${ }^{4}$
Abstract

Objective To evaluate and compare the diagnostic accuracy of the Heidelberg retina tomograph (HRT) version 3 with that of glaucoma specialists using stereophotography in discriminating between normal eyes and patients with early glaucomatous visual field loss.

Methods A total of $\mathbf{1 0 5}$ eyes of $\mathbf{1 0 5}$ individuals were prospectively and consecutively recruited. The sample comprised 51 normal and 54 early glaucomatous eyes, as defined by intraocular pressure and standard automated perimetry results, regardless of optic disc appearance. Receiver operating characteristic (ROC) curves were plotted for the HRT3 parameters and a linear discriminant function (LDF) developed in our hospital. Best sensitivity-specificity pairs were compared between the HRT3 parameters, with the highest areas under the ROC curve (AUCs) and evaluation of optic disc stereophotographs. Agreement between methods for measuring vertical cup-to-disc ratio was evaluated with the Bland-Altman plot.

Results The average visual field mean deviation was $-2.90 \mathrm{~dB}$. The HRT3 parameters with the largest AUCs were our LDF (0.900), rim volume (0.883), and vertical cup/disk ratio $(0.880)$, with no significant differences between these parameters. Sensitivity-specificity pairs were $79.6-100 \%$ (clinical evaluation), $83.3-86.3 \%$ (our LDF), 64.8-96.1\% (final glaucoma probability score), and $68.5-90.2 \%$ (global Moorfields regression analysis).
Conclusions The diagnostic accuracy for differentiating normal eyes from those with early visual field defects was similar between clinical evaluation of the optic disc and evaluation with the HRT3. The use of our LDF increased the sensitivity-specificity balance with respect to the HRT-provided parameters. The diagnostic accuracy of the HRT classifications was comparable to that of an experienced glaucoma specialist.

Eye (2010) 24, 123-130; doi:10.1038/eye.2009.14; published online 13 February 2009

Keywords: glaucoma; diagnosis; optic disc; Heidelberg retina tomograph; HRT; stereophotographs

\section{Introduction}

The natural evolution of primary open-angle glaucoma implies the loss of ganglion cells and their axons in the retina. ${ }^{1,2}$ Damage to the retinal nerve fibre layer (RNFL) is usually followed by changes in the optic nerve head $(\mathrm{ONH})$ and typical visual field defects. Because of the wide variability of normal optic disc morphology, a definitive glaucoma diagnosis based on the ONH morphology often depends on the observer's experience and is therefore quite subjective. Thus, during the last several years, different automatic diagnosis techniques have been developed. One of these methods is the Heidelberg retina tomograph (HRT), which provides quantitative and objective measures of the optic disc and the peripapillary RNFL. The latest version of the HRT software contains the Advanced Glaucoma Analysis 3.0. HRT3
${ }^{1}$ Department of Ophthalmology, Miguel Servet University Hospital, Instituto Aragonés de Ciencias de la Salud, Zaragoza, Spain

${ }^{2}$ G.B. Bietti FoundationIRCCS (Istituto di Ricovero

e Cura a Carattere Scientifico), Rome, Italy

${ }^{3}$ Ophthalmology, Department of Neuroscience, University of Pisa, Italy

${ }^{4}$ Sanitas residencial, Zaragoza, Spain

Correspondence: A Ferreras, Department of Ophthalmology, Miguel Servet University Hospital,

Isabel la Católica 1-3, Zaragoza 50009, Spain

Tel: + 34 976765558;

Fax: + 34976566234

Email: aferreras@

msn.com

Received: 20 October 2008 Accepted in revised form:

4 January 2009

Published online: 13

February 2009

Conflict of interest: None 
provides larger, ethnic-selectable normative databases, and includes new data analysis tools such as the Glaucoma Probability Score (GPS). ${ }^{3,4}$ Most HRT3 analyses require prior manual outlining of disc boundaries. The GPS, however, does not rely on reference planes and does not need for a contour line drawing, reducing the dependency on operator skill.

The ability of the HRT to detect glaucomatous changes has been extensively validated..$^{5-9}$ Nevertheless, the accuracy of HRT for glaucoma diagnosis should be compared with that of clinical $\mathrm{ONH}$ evaluation by stereophotography, which is considered the reference standard for assessing optic disc damage, ${ }^{10}$ to clarify the advantages of this new device.

Several factors threaten the internal and external validity of a study of diagnostic accuracy, a fact that inspired the launch of the Standards for Reporting of Diagnostic Accuracy (STARD) initiative. ${ }^{11}$ The objective of the STARD initiative is to improve the quality of the reporting of studies of diagnostic accuracy. Shunmugan and Azuara-Blanco ${ }^{12}$ evaluated the quality of the reporting of diagnostic accuracy using the HRT and found that it was suboptimal. Thus, they suggested the STARD initiative to improve the quality of these studies. The design of the present study followed all 25 items of the STARD guidelines.

The objective of this study was to evaluate and compare the diagnostic ability of optic disc stereophotography assessment by a glaucoma specialist and the HRT3 to discriminate between normal eyes and eyes with mild glaucomatous defects in standard automated perimetry (SAP). To our knowledge, this is the first study to perform this comparison. Our case-sample consisted of a group of patients with high intraocular pressure (IOP) and mild SAP defects.

\section{Materials and methods}

\section{Subjects and measurement protocol}

The study protocol adhered to the tenets of the Declaration of Helsinki for humans in biomedical research. The ethics committee of our hospital approved the study and written informed consent was obtained from all participants.

\section{Subjects}

From January 2008 to June 2008, 118 consecutive subjects were prospectively pre-selected from two outpatient clinics under the area of influence of the Miguel Servet University Hospital, Zaragoza, Spain.
Normal eyes were recruited from patients referred for refraction that underwent routine examination without abnormal ocular findings, hospital staff, and relatives of patients in our hospital. Inclusion criteria for all patients were best corrected visual acuity of $20 / 30$ or better, refractive error not exceeding 5.00 diopters sphere and/ or 2.00 diopters cylinder, no previous intraocular surgery, age over 18 years, and transparent ocular media (nuclear colour/opalescence, cortical or posterior subcapsular lens opacity $<1$ ) according to the Lens Opacities Classification System III system. ${ }^{13}$ The exclusion criteria were previous intraocular surgery, diabetes or other systemic diseases, history of ocular or neurologic disease, current use of a medication that could affect visual field sensitivity, or visual field losses greater than mild defects according to the Hodapp-Parrish-Anderson score. ${ }^{14}$ Initial damage in SAP was defined as a mean deviation greater than $-6 \mathrm{~dB}$; or less than 18 points lower than the $5 \%$ probability level in the pattern plot; and/or less than 10 points lower than $1 \%$ and an absence of points lower than $5 \%$ in the central 10 degrees, and no points lower than $15 \mathrm{~dB}$ at any location.

Two of the pre-selected subjects did not provide informed consent and six subjects did not complete all of the required tests. In five subjects, GPS analyses produced only a global result or no results, because the GPS model was partially or completely incompatible with the shape of the ONH. All of these subjects were excluded from further analysis. Finally, 105 subjects of Caucasian origin were included in the study. One eye from each subject was randomly chosen, unless only one eye met the inclusion criteria.

All participants underwent a full ophthalmologic examination including biomicroscopy, gonioscopy, Goldmann applanation tonometry, pachymetry (model DGH 500; DGH Technology, Exton, PA, USA), and ophthalmoscopy of the posterior segment. At least two reliable SAP tests were performed using a Humphrey Field analyzer, model 750i (Humphrey Field Analyzer; Carl Zeiss Meditec, Dublin, CA, USA), with the SITA Standard 24-2 program. Near addition was added to the subject's refractive correction. If fixation losses and false positive or false negative rates were greater than $20 \%$, the test was repeated. The last reliable perimetry test obtained was used in the analysis to minimise the learning effect. ${ }^{15}$ Abnormal $\mathrm{SAP}$ results were considered to have a reproducible glaucomatous visual field loss in the absence of any other abnormalities to explain the defect. Visual field loss was defined as a pattern standard deviation significantly above the 5\% level and/or a Glaucoma Hemifield Test outside normal limits. The subjects completed the perimetry tests before undergoing any clinical examination or structural test. Each SAP test was performed on different days to avoid inducing a fatigue effect. 
Clinical assessment of the $\mathrm{ONH}$ was performed after mydriasis (0.5\% tropicamide; Alcon Laboratories Inc., Fort Worth, TX, USA) by evaluating stereophotographs of the optic disc (Canon CF-60UV fundus camera; Canon Inc., Tokyo, Japan). Glaucomatous damage was defined as focal or diffuse neuroretinal rim narrowing with concentric enlargement of the optic cup, localised notching, or both. ${ }^{16}$ Two glaucoma specialists masked to patient identity and clinical history evaluated the photographs using a stereoscopic viewer and classified the eyes as glaucomatous or normal. The stereophotographs were examined independently and any disagreement was resolved by consensus.

Topographic analysis of the optic disc was performed using the HRT3 (Heidelberg Engineering, Dossenheim, Germany), which provides topographic measurements of the ONH derived from 16 to 64 optical sections to a depth of $4 \mathrm{~mm}$, depending on the longitudinal field of view. ${ }^{17}$ The spherical equivalent refractive error of each eye was adjusted in the dioptric ring of the HRT. Magnification errors were corrected by the software based on keratometric readings. Topographic images were then obtained through dilated pupils ( $0.5 \%$ tropicamide) and analysed using the Advanced Glaucoma Analysis 3.0 software. All scans had to have an interscan standard deviation of less than $30 \mu \mathrm{m}$. The margin of the optic discs was manually traced by the same glaucoma specialist, who was masked to the patients' identity and clinical history, defining the inner edge of the Elschnig's ring with at least a four-point contour line. The global stereometric parameters investigated in this study were cup area, rim area, cup/disc area ratio, rim/disc area ratio, cup volume, rim volume, mean cup depth, maximum cup depth, height variation contour, cup shape measure, mean RNFL thickness, RNFL cross-section area, horizontal cup/disk ratio, vertical cup/disk ratio, maximum contour elevation, maximum contour depression, contour line modulation temporal-superior, contour line modulation temporal-inferior, average variability, reference height, FSM (FS Mikelberg) discriminant function value, ${ }^{18}$ and RB (RO Burk $)^{19}$ discriminant function value. We also evaluated a linear discriminant function (LDF) developed in our hospital, ${ }^{20}$ and GPS $^{3,4}$ and Moorfields regression analysis (MRA) classifications. ${ }^{6}$ Both, GPS and MRA, provided a global classification (derived for the entire image area), sector classifications (disease probability scores for each of the six sectors in which the optic disc is divided), and a final classification (an overall result based on the combination of both sector and global results).

All ophthalmic examinations, perimetry tests, and the topographic analysis were performed within 6 weeks of the subject's date of enrollment into the study.

\section{Classification into groups}

Healthy eyes had an IOP of less than $21 \mathrm{mmHg}$, no history of increased IOP, and a normal SAP.

Glaucomatous eyes had an IOP greater than $21 \mathrm{mmHg}$ (on at least three readings on different days) and typical SAP defects, regardless of the appearance of the optic disc.

\section{Statistical analyses}

All statistical analyses were performed using SPSS (version 15.0; SPSS Inc., Chicago, IL, USA) and MedCalc (version 9.6.4.0 MedCalc Software, Mariakerke, Belgium) statistical software. Differences between groups were assessed using a two-tailed Student's $t$-test. A $P$-value of less than 0.05 was considered to be statistically significant. First, the Kolmogorov Smirnov test was applied to check that the data were normally distributed. The receiver operating characteristic (ROC) curves were plotted between normal and glaucomatous eyes for all global HRT3 stereometric parameters and our LDF. The ROC curve has a trade-off between sensitivity and 1-specificity (false positive rate). An area under the ROC curve (AUC) of 1.0 represents perfect discrimination, whereas an AUC of 0.5 represents chance discrimination. ROC curves were used to assess the usefulness of each parameter for differentiating glaucomatous eyes from healthy eyes. Differences between the AUCs were tested using the Hanley-McNeil method. ${ }^{21}$

Sensitivities at $95 \%$ (5\% false positive rate) fixed specificity were calculated for the HRT3 parameters. The diagnostic ability of the final and global colour-coded MRA and GPS classifications were also calculated. The cutoff points were defined by the MedCalc software as the points with the best sensitivity-specificity balance. Positive and negative likelihood ratios (LRs) were calculated for these classifications. ${ }^{22}$ A positive LR is the ratio between the probability of a positive test result given the presence of the disease and the probability of a positive test result given the absence of the disease (sensitivity/1-specificity) or true positive rate/false positive rate). The LR for a given test result indicates how much that result increases or decreases the post-test probability of disease. ${ }^{23} \mathrm{~A}$ negative LR is the ratio between the probability of a negative test result given the presence of the disease and the probability of a negative test result given the absence of the disease (1-sensitivity/specificity or false negative rate/true negative rate). An LR value of 1 indicates that the test provides no additional information, but ratios higher or lower than 1 indicate an increased or decreased post-test probability of disease, respectively. 
After confirming that the values for each of the variables were normally distributed, Pearson correlations were calculated between the vertical cup-to-disc ratio evaluated in stereophotographs and the vertical cup/disk ratio measured with the HRT3 in the total sample and in normal and glaucomatous populations.

To determine the agreement between the vertical cup-to-disc ratio evaluated in stereophotographs and the vertical cup/disk ratio measured with the HRT, a Bland-Altman plot was produced. The Bland and Altman plot $^{24,25}$ is a statistical method used to assess agreement between two measurement techniques. This graphical method was used to plot the differences between clinical and HRT ONH assessments against the averages of both techniques. Horizontal lines were drawn at the mean difference, and at the mean difference plus and minus 1.96 times the standard deviation of the differences. If the differences within the mean \pm 1.96 standard deviations are not clinically important, the two methods may be used interchangeably. The plot is useful for revealing the relationship between the differences and the averages and is useful for identifying systematic biases and possible outliers.

\section{Results}

The Kolmogorov Smirnov test confirmed that all variables analysed in this study had a normal distribution. Table 1 shows the clinical characteristics of both groups included in the study. The normal group consisted of 51 eyes of 51 healthy individuals (mean age $59.1 \pm 9.6$ years). The glaucoma group included 54 eyes of 54 patients (mean age $61.9 \pm 6.8$ years): 43 with primary open-angle glaucoma, 10 with pseudoexfoliative glaucoma, and 1 with pigmentary glaucoma. Age $(P=0.087)$, best corrected visual acuity $(P=0.168)$, central corneal thickness $(P=0.342)$, and disc area measured with the HRT3 $(P=0.090)$ did not differ significantly between the groups.

The HRT3 parameters with the greatest AUCs (Table 2) were our LDF, rim volume, and vertical cup/disk ratio. There were no significant differences between the AUCs for these three parameters (Figure 1). At a fixed specificity of $95 \%$, our LDF, and the FSM and RB discriminant functions had the best sensitivity values. The parameters with best sensitivity-specificity pairs were clinical evaluation of the ONH, our LDF, final GPS and global MRA (Table 3). Final and global GPS had the highest positive LRs, whereas rim volume and vertical cup/disk ratio showed the lowest negative LRs.

In the total sample, vertical cup-to-disc ratio evaluated on stereophotographs strongly correlated with the vertical cup/disk ratio measured with the HRT3 (0.845; $P<0.001)$. This correlation was $0.709(P<0.001)$ in the normal group and $0.807(P=0.001)$ in the glaucoma group.

The Bland-Altman plot (Figure 2) did not reveal a significant difference between measurements of the vertical cup-to-disc ratio with stereophotographs and HRT3 (slope, $0.0056 \pm 0.05 ; P=0.918$ ). Only in five cases $(4.8 \%)$ was the difference between both methods greater than 1.96 standard deviations from the mean.

\section{Discussion}

To minimise glaucomatous damage, the recognition of optic disc changes at an early stage of the disease is a key point. Numerous studies ${ }^{5-9,26}$ have been performed to determine the best HRT parameters for discriminating between healthy and glaucoma subjects. Others studies ${ }^{18-20,27}$ have focused on developing discriminant functions to improve the diagnostic ability of HRT parameters. Here, we compared the usefulness and accuracy of single and combined parameters, LDFs, of

Table 1 Demographic and clinical characteristics of the study sample

\begin{tabular}{|c|c|c|c|}
\hline & Control group $(\mathrm{n}=51)$ & Glaucoma group $(\mathrm{n}=54)$ & P-value \\
\hline & Mean (SD) & Mean $(S D)$ & \\
\hline Age (years) & $59.08(9.58)$ & $61.87(6.84)$ & $0.087^{\mathrm{a}}$ \\
\hline BCVA (Snellen) & $0.89(0.10)$ & $0.86(0.12)$ & $0.168^{\mathrm{a}}$ \\
\hline Mean IOP (mmHg) & $14.57(2.24)$ & $23.74(3.57)$ & $<0.001^{\mathrm{a}}$ \\
\hline Pachymetry $(\mu \mathrm{m})$ & $548.9(27.11)$ & $555.0(35.08)$ & $0.342^{\mathrm{a}}$ \\
\hline $\mathrm{CD}$ & $0.32(0.16)$ & $0.67(0.19)$ & $<0.001^{\mathrm{a}}$ \\
\hline MD of SAP (dB) & $-1.13(2.27)$ & $-2.91(2.28)$ & $<0.001^{\mathrm{a}}$ \\
\hline PSD of SAP & $1.52(1.83)$ & $3.16(2.07)$ & $<0.001^{\mathrm{a}}$ \\
\hline HRT3 disc area $\left(\mathrm{mm}^{2}\right)$ & $1.94(0.31)$ & $2.06(0.40)$ & $0.090^{\mathrm{a}}$ \\
\hline
\end{tabular}

$\mathrm{SD}$, standard deviation; $\mathrm{BCVA}$, best corrected visual acuity; IOP, basal intraocular pressure (without treatment); $\mathrm{CD}$, vertical cup-to-disc ratio in stereophotographs; MD, mean deviation; SAP, standard automated perimetry; PSD, pattern standard deviation; HRT, Heidelberg retina tomograph.

a'Student's $t$-test between control and glaucoma patients. 
Table 2 Areas under the receiver operating characteristic curve and sensitivities at 95\% fixed specificity for each global topographic parameter obtained with the Heidelberg retina tomograph 3

\begin{tabular}{|c|c|c|c|c|c|c|}
\hline \multirow[t]{2}{*}{ HRT3 global topographic parameters } & \multirow[t]{2}{*}{ AUC } & \multirow[t]{2}{*}{$S D$} & \multicolumn{2}{|c|}{$95 \% \mathrm{CI}$} & \multirow[t]{2}{*}{ P-value } & \multirow{2}{*}{$\begin{array}{l}\text { Sensitivity at } 95 \% \\
\text { fixed specificity }\end{array}$} \\
\hline & & & Lower bound & Upper bound & & \\
\hline LDF & 0.900 & 0.031 & 0.841 & 0.960 & $<0.001$ & 75.9 \\
\hline RB discriminant function value & 0.872 & 0.036 & 0.802 & 0.943 & $<0.001$ & 66.7 \\
\hline FSM discriminant function value & 0.869 & 0.034 & 0.801 & 0.936 & $<0.001$ & 66.7 \\
\hline Vertical cup/disk ratio & 0.880 & 0.034 & 0.814 & 0.946 & $<0.001$ & 63.0 \\
\hline Linear cup/disc ratio & 0.861 & 0.037 & 0.789 & 0.934 & $<0.001$ & 63.0 \\
\hline Cup/disc area ratio & 0.861 & 0.037 & 0.787 & 0.934 & $<0.001$ & 63.0 \\
\hline Rim/disc area ratio & 0.861 & 0.037 & 0.787 & 0.934 & $<0.001$ & 63.0 \\
\hline Rim area & 0.854 & 0.036 & 0.783 & 0.925 & $<0.001$ & 59.3 \\
\hline Maximum contour elevation & 0.802 & 0.043 & 0.718 & 0.887 & $<0.001$ & 55.6 \\
\hline Rim volume & 0.883 & 0.032 & 0.819 & 0.946 & $<0.001$ & 50.0 \\
\hline Cup shape measure & 0.808 & 0.042 & 0.727 & 0.890 & $<0.001$ & 48.2 \\
\hline GPS global & 0.822 & 0.044 & 0.713 & 0.886 & $<0.001$ & 46.3 \\
\hline Cup area & 0.826 & 0.041 & 0.745 & 0.906 & $<0.001$ & 44.4 \\
\hline CLM temporal-inferior & 0.802 & 0.042 & 0.719 & 0.884 & $<0.001$ & 42.6 \\
\hline CLM temporal-superior & 0.804 & 0.042 & 0.722 & 0.886 & $<0.001$ & 36.0 \\
\hline Mean RNFL thickness & 0.852 & 0.038 & 0.777 & 0.927 & $<0.001$ & 33.3 \\
\hline Horizontal cup/disk ratio & 0.784 & 0.045 & 0.696 & 0.872 & $<0.001$ & 33.3 \\
\hline RNFL cross-sectional area & 0.843 & 0.039 & 0.766 & 0.919 & $<0.001$ & 27.8 \\
\hline Cup volume & 0.763 & 0.047 & 0.670 & 0.856 & $<0.001$ & 24.1 \\
\hline Maximum cup depth & 0.541 & 0.058 & 0.428 & 0.654 & 0.471 & 18.5 \\
\hline Height variation contour & 0.771 & 0.047 & 0.680 & 0.863 & $<0.001$ & 16.7 \\
\hline Disc area & 0.604 & 0.055 & 0.495 & 0.712 & 0.067 & 7.4 \\
\hline Mean cup depth & 0.654 & 0.054 & 0.549 & 0.759 & 0.006 & 7.4 \\
\hline Maximum contour depression & 0.574 & 0.056 & 0.464 & 0.684 & 0.192 & 7.4 \\
\hline Average variability & 0.564 & 0.056 & 0.454 & 0.675 & 0.255 & 5.6 \\
\hline Reference height & 0.513 & 0.057 & 0.401 & 0.625 & 0.815 & 0 \\
\hline
\end{tabular}

HRT, Heidelberg retina tomography; AUC, area under the receiver operating characteristic curve; SD, standard deviation; CI, confidence interval; RNFL, retinal nerve fibre layer; CLM, contour line modulation; GPS, glaucoma probability score; LDF, linear discriminant function.

Parameters are ranked in descending order of sensitivity.

the HRT3 with observations by an experienced ophthalmologist in patients with initial visual field defects for detecting glaucomatous changes.

The design of our study included a single homogeneous population for the comparison of two different diagnostic methods. In our population sample, disc areas were similar between the normal and glaucoma groups, which is a critical factor when comparing groups because several HRT parameters are directly related to disc size. ${ }^{5,27}$ MRA and GPS are also disc size dependent, ${ }^{4,9}$ and tend to classify small and large optic discs with lower sensitivity and greater specificity than medium-sized discs. Clinicians must take disc size into account when interpreting HRT outcomes. $^{5,28}$

ROC curves were only plotted for the global stereometric parameters and global GPS numerical values because the MRA and colour-coded GPS classifications are not continuous variables. The small number of categories in these cases might lead to an underestimation of the AUC. ${ }^{29}$
The optic disc morphology correlated with findings from the tests used for evaluation (stereophotographs and HRT); therefore, the groups were classified regardless of optic disc appearance to avoid an overestimation of the sensitivity and specificity of the variables. ${ }^{30,31}$

In general, a difference of up to $4 \%$ can be expected between data obtained with the previous HRT version (HRT2) compared with HRT3. A horizontal scaling error in the HRT2 reported by the manufacturer, in which the horizontal stereometric measurements were increased by $4 \%$, was corrected in the HRT3. ${ }^{32}$ The new v3.0 software for the HRT glaucoma module has enhanced alignment algorithms and therefore has somewhat better performance for glaucoma diagnosis. ${ }^{33}$

Traditionally, physician drawings and photographic documentation of the $\mathrm{ONH}$ have been the bases of glaucoma management. ONH evaluation is subjective, however, and stereophotographs are not always taken in clinical practice. Thus, there is a low rate of compliance with the recommendations for $\mathrm{ONH}$ documentation in 
the preferred practice patterns for glaucoma. ${ }^{34-37}$ Fremont $e a^{36}$ found that almost half of glaucoma patients did not have a photograph or drawing of the $\mathrm{ONH}$ made at their first evaluation. Although $\mathrm{ONH}$ evaluation is relatively easy to perform using a slit-lamp with a bi-convex lens, interpreting and recording the observed data may be more difficult. The HRT provides objective and quantitative measurements that are highly reproducible and easier to obtain than

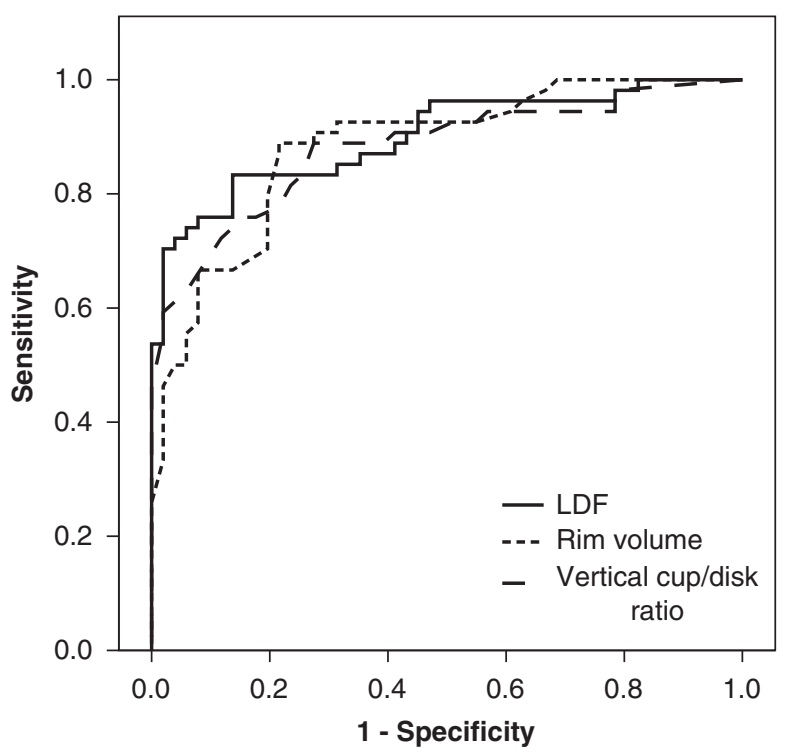

Figure 1 Receiver operating characteristic (ROC) curves of the HRT3's parameters with the largest areas under the receiver operating characteristic curve. Area under the ROC curve (AUC) for the linear discriminant function (LDF): 0.900 (95\% confidence interval: 0.841-0.960); AUC for rim volume: $0.883(95 \%$ confidence interval: 0.819-0.946), and AUC for RB: 0.880 (95\% confidence interval: $0.814-0.946$ ). stereophotographs. Moreover, HRT may be useful as a first objective diagnostic approach and provides baseline data for detecting changes in the follow-up examinations. Non-quantitative changes, however, such as disc hemorrhage, optic disc pallor, parapapillary atrophy, or RNFL defects, cannot be measured with this imaging technology.

In the present study, the ability to discriminate between healthy and glaucomatous eyes was similar for the glaucoma specialist and the HRT3. Subjective evaluation of stereophotographs and HRT3 showed good agreement in measuring the vertical cup-to-disc ratio. Thus, based on Bland-Altman plot results and the strong correlations between the two methods, these methods can be used interchangeably in clinical practice.

Nevertheless, the vertical cup-to-disc ratio is highly dependent on optic disc size. ${ }^{38}$ Large optic discs have physiologically increased cupping that may be confused with glaucoma, and conversely, small glaucomatous discs may not show any perceptible cupping or neural rim atrophy. Therefore, the disc size should always be considered when evaluating the ONH.

The LDFs, our $\mathrm{LDF}^{20}$ and the HRT-provided LDFs $\left(\mathrm{FSM}^{18}\right.$ and $\left.\mathrm{RB}^{19}\right)$, were the parameters with the highest sensitivities at a $95 \%$ fixed specificity. The LDFs represent a combination of variables whose value is as similar as possible within groups and as different as possible between groups. Therefore, we suggest that LDFs, or even a combination of learning classifiers, be used to increase the diagnostic ability of single HRT variables.

In our study, the clinical ONH evaluation was very conservative (100\% specificity). GPS and global MRA classifications, however, also had a very high specificity at early stages of the disease. GPS exhibited higher specificities (and the highest negative LR) and somewhat

Table 3 Best pairs of sensitivity-specificity (defined by MedCalc software), and likelihood ratios of Moorfields regression analysis and glaucoma probability score classifications to discriminate between normal and glaucomatous eyes

\begin{tabular}{|c|c|c|c|c|c|}
\hline & Cutoff point & Sensitivity (\%) & Specificity (\%) & $+L R$ & $-L R$ \\
\hline \multicolumn{6}{|l|}{ HRT3 parameter } \\
\hline LDF & $>0.0371$ & 83.3 & 86.3 & 6.07 & 0.19 \\
\hline Rim volume & $\leqslant 0.33$ & 88.9 & 78.4 & 4.12 & 0.14 \\
\hline Vertical cup/disk ratio & $>0.49$ & 88.9 & 72.5 & 3.24 & 0.15 \\
\hline \multicolumn{6}{|l|}{ Colour-coded cutoff points } \\
\hline Final MRA & Borderline & 85.2 & 70.6 & 2.89 & 0.20 \\
\hline Global MRA & Borderline & 68.5 & 90.2 & 6.98 & 0.34 \\
\hline Final GPS & ONL & 64.8 & 96.1 & 16.61 & 0.36 \\
\hline Global GPS & ONL & 55.6 & 96.1 & 14.25 & 0.46 \\
\hline $\mathrm{CD}$ & 0.55 & 74.1 & 92.2 & 9.5 & 0.28 \\
\hline Final ophthalmologist's evaluation & - & 79.6 & 100 & - & 0.20 \\
\hline
\end{tabular}

LR, likelihood ratio; HRT, Heidelberg retina tomograph; MRA, Moorfields regression analysis; GPS, glaucoma probability score; ONL, outside normal limits; CD, vertical cup-to-disc ratio in stereophotographs.

The clinical diagnostic ability of an experienced ophthalmologist is also shown below. 


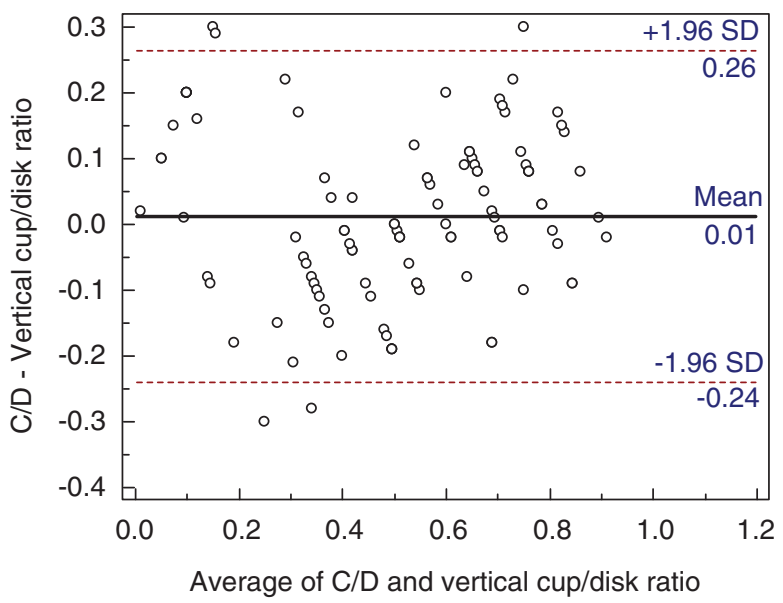

Figure 2 Bland-Altman plot of cup-to-disc ratio and vertical cup/disk ratio evaluated with stereophotographs and Heidelberg retina tomograph, respectively.

lower sensitivity than MRA classification, which indicates that an abnormal GPS result is associated with a big change in the post-test probability of disease, and therefore has a greater ability to confirm the presence of glaucoma. Rim volume and the vertical cup/disk ratio had the lowest negative LRs, and therefore were the most accurate parameters for excluding the presence of glaucoma.

Differences in study design and visual field loss severity make it difficult to compare results among studies. Obviously, visual field loss severity strongly influences imaging instrument sensitivity. ${ }^{9,28}$ Our sample comprised individuals with mild visual field defects, but more severe disease would be expected to be associated with increased sensitivity.

In four (7.8\%) control subjects and one (1.8\%) patient with glaucoma, the GPS was unable to provide a classification, and these cases were excluded from our study. Coops $e t \mathrm{al}^{4}$ suggested that in normal individuals, the failure of GPS to match the surface of the optic disc occurred most often in small and crowded discs, but they could not identify a single distinct cause of failure in the glaucoma group. The quality of the data obtained by the imaging devices is influenced by the media opacity, retinal pigment epithelium status, instrument variability, and positioning and centering of the images, and therefore, these factors should be taken into account when assessing HRT. Moreover, in clinical practice, the interpretation of disc size or changes in the ONH based on stereophotographs may be difficult for discs with atypical morphology. In these cases, the performance of both methods may be worse.

Our results confirm the usefulness of HRT3 for early glaucoma diagnosis, and indicate that the performance of this device in diagnosing glaucomatous changes is similar to that of a well-trained ophthalmologist. In other words, a technician using HRT3 may achieve the diagnostic accuracy of an expert when dealing with these difficult cases. These results are consistent with those of other studies, ${ }^{39-42}$ in which the HRT2 was reported to have a similar sensitivity for diagnosing glaucoma as clinical assessment using stereoscopic optic disc photographs, and provide validation for the HRT3.

\section{References}

1 Quigley HA. Neuronal death in glaucoma. Prog Retin Eye Res 1999; 18: 39-57.

2 American Academy of Ophthalmology Glaucoma Panel. Preferred Practice Pattern. Primary open-angle glaucoma. American Academy of Ophthalmology: San Francisco, CA, 2005, p3.

3 Swindale NV, Stjepanovic G, Chin A, Mikelberg FS. Automated analysis of normal and glaucomatous optic nerve head topography images. Invest Ophthalmol Vis Sci 2000; 41: 1730-1742.

4 Coops A, Henson DB, Kwartz AJ, Artes PH. Automated analysis of Heidelberg retina tomograph optic disc images by glaucoma probability score. Invest Ophthalmol Vis Sci 2006; 47: 5348-5355.

5 Iester M, Mikelberg FS, Drance SM. The effect of optic disc size on diagnostic precision with the Heidelberg retina tomograph. Ophthalmology 1997; 104: 545-548.

6 Wollstein G, Garway-Heath DF, Hitchings RA. Identification of early glaucoma cases with the scanning laser ophthalmoscope. Ophthalmology 1998; 105: 1557-1563.

7 Zangwill LM, Bowd C, Berry CC, Williams J, Blumenthal EZ, Sánchez-Galeana CA et al. Discriminating between normal and glaucomatous eyes using the Heidelberg retina tomograph, GDx nerve fiber analyzer, and optical coherence tomograph. Arch Ophthalmol 2001; 119: 985-993.

8 Medeiros FA, Zangwill LM, Bowd C, Weinreb RN. Comparison of the GDx VCC scanning laser polarimeter, HRT II confocal scanning laser ophthalmoscope, and Stratus OCT optical coherence tomograph for the detection of glaucoma. Arch Ophthalmol 2004; 122: 827-837.

9 Ferreras A, Pajarín AB, Polo V, Larrosa JM, Pablo LE, Honrubia FM. Diagnostic ability of Heidelberg retina tomograph 3 classifications: glaucoma probability score versus Moorfields regression analysis. Ophthalmology 2007; 114: 1981-1987.

10 Sommer A, Pollack I, Maumenee AE. Optic disc parameters and onset of glaucomatous field loss. II. Static screening criteria. Arch Ophthalmol 1979; 97: 1449-1454.

11 Bossuyt PM, Reitsma JB, Bruns DE, Gatsonis CA, Glasziou PP, Irwig LM et al. The STARD statement for reporting studies for diagnostic accuracy: explanation and elaboration. Clin Chem 2003; 49: 7-18.

12 Shunmugan M, Azuara-Blanco A. The quality of reporting of diagnostic accuracy studies in glaucoma using the Heidelberg retina tomograph. Invest Ophthalmol Vis Sci 2006; 47: 2317-2323.

13 Chylack Jr LT, Wolfe JK, Singer DM, Leske MC, Bullimore MA, Bailey IL et al. Longitudinal study of cataract study 
group. The lens opacities classification system III. Arch Ophthalmol 1993; 111: 831-836.

14 Hodapp E, Parrish II RK, Anderson DR. Clinical Decisions in Glaucoma. Mosby: St Louis, MO, 1993, pp 52-61.

15 Heijl A, Lindgren A, Lindgren G. Test-retest variability in glaucomatous visual fields. Am J Ophthalmol 1989; 108: 130-135.

16 Tuulonen A, Airaksinen PJ. Initial glaucomatous optic disk and retinal nerve fiber layer abnormalities and their progression. Am J Ophthalmol 1991; 111: 485-490.

17 Girkin CA. Principles of confocal scanning laser ophthalmoscopy for the clinician. In: Fingeret M, Flanagan JG, Liebmann JM (eds). The Essential HRT Primer. Heidelberg Engineering: Heidelberg, Germany, 2005, pp 1-9.

18 Mikelberg FS, Parfitt CM, Swindale NV, Graham SL, Drance SM, Gosine R. Ability of the Heidelberg retina tomograph to detect early glaucomatous visual field loss. J Glaucoma 1995; 4: 242-247.

19 Burk RO, Noack H, Rohrschneider K, Volcker HE. Prediction of glaucomatous visual fields defects by reference plane independent three-dimensional optic nerve head parameters. In: Wall M, Wild JM (eds). Perimetry Update 1998/99: Proceedings of the XIII International Perimetric Society Meeting Gardone Riviera (BS), 1998. Walter Kugler: The Hague, 1999. pp 463-474.

20 Ferreras A, Pablo LE, Larrosa JM, Polo V, Pajarín AB, Honrubia FM. Discriminating between normal and glaucoma-damaged eyes with the Heidelberg retina tomograph 3. Ophthalmology 2008; 115: 775-781.

21 Hanley JA, McNeil BJ. A method of comparing the areas under receiver operating characteristic curves derived from the same cases. Radiology 1983; 148: 839-843.

22 Garway-Heath DF, Friedman DS. How should results from clinical tests be integrated into the diagnostic process? Ophthalmology 2006; 113: 1479-1480.

23 Radack KL, Rouan G, Hedges J. The likelihood ratio: an improved measure for reporting and evaluating diagnostic test results. Arch Pathol Lab Med 1986; 110: 689-693.

24 Bland JM, Altman DG. Statistical methods for assessing agreement between two methods of clinical measurement. Lancet 1986; 1: 307-310.

25 Dewitte K, Fierens C, Stöckl D, Thienpont LM. Application of the Bland-Altman plot for interpretation of methodcomparison studies: a critical investigation of its practice. Clin Chem 2002; 48: 799-801.

26 Oddone F, Centofanti M, Rossetti L, Iester M, Fogagnolo P, Capris E et al. Exploring the Heidelberg retinal tomograph 3 diagnostic accuracy across disc sizes and glaucoma stages: a multicenter study. Ophthalmology 2008; 115: 1358-1365.

27 Bathija R, Zangwill L, Berry CC, Sample PA, Weinreb RN. Detection of early glaucomatous structural damage with confocal scanning laser tomography. J Glaucoma 1998; 7: $121-127$.
28 Medeiros FA, Zangwill LM, Bowd C, Sample PA, Weinreb RN. Influence of disease severity and optic disc size on the diagnostic performance of imaging instruments in glaucoma. Invest Ophthalmol Vis Sci 2006; 47: 1008-1015.

29 Wagner RR, Beiden SV, Metz CE. Continuous versus categorical data for ROC analysis: some quantitative considerations. Acad Radiol 2001; 8: 328-334.

30 Garway-Heath DF, Hitchings RA. Sources of bias in studies of optic disc and retinal nerve fibre layer morphology. Br J Ophthalmol 1998; 82: 986.

31 Medeiros FA. How should diagnostic tests be evaluated in glaucoma? Br J Ophthalmol 2007; 91: 273-274.

32 Heidelberg Retina Tomograph Glaucoma Module. Operating Instructions Software Version 3.0. Heidelberg Engineering: Heidelberg, Germany, 2005.

33 Ferreras A, Pablo LE, Pajarín AB, Larrosa JM, Polo V, Pueyo V. Diagnostic ability of the Heidelberg retina tomograph 3 for glaucoma. Am J Ophthalmol 2008; 145: 354-359.

34 Hertzog LH, Albrecht KG, LaBree L, Lee PP. Glaucoma care and conformance with preferred practice patterns: examination of the private, community-based ophthalmologist. Ophthalmology 1996; 103: 1009-1013.

35 Shingleton BJ, Crandall A, Johnstone M, Robin A, Brown R. Medical treatment patterns of ASCRS members for primary open-angle glaucoma: 1998 survey. J Cataract Refract Surg 1999; 25: 118-127.

36 Fremont AM, Lee PP, Mangione CM, Kapur K, Adams JL, Wickstrom SL et al. Patterns of care for open-angle glaucoma in managed care. Arch Ophthalmol 2003; 121: 777-783.

37 American Academy of Ophthalmology Glaucoma Panel. Preferred Practice Pattern. Primary open-angle glaucoma. American Academy of Ophthalmology: San Francisco, CA, 2005, p 11.

38 Garway-Heath D, Ruben ST, Viswanathan A, Hitchings RA. Vertical cup/disc ratio in relation to optic disc size: its value in the assessment of the glaucoma suspect. Br J Ophthalmol 1998; 82: 1118-1124.

39 Wollstein G, Garway-Heath DF, Fontana L, Hitchings RA. Identifying early glaucomatous changes. Ophthalmology 2000; 107: 2272-2277.

40 Bowd C, Zangwill L, Medeiros FA, Hao J, Chan K, Lee TW. et al. Confocal scanning laser ophthalmoscopy classifiers and stereophotograph evaluation for prediction of visual field abnormalities in glaucoma-suspect eyes. Invest Ophthalmol Vis Sci 2004; 45: 2255-2262.

41 Deleon-Ortega JE, Arthur SN, McGwin Jr G, Xie A, Monheit BE, Girkin CA. Discrimination between glaucomatous and nonglaucomatous eyes using quantitative imaging devices and subjective optic nerve head assessment. Invest Ophthalmol Vis Sci 2006; 47: 3374-3380.

42 Medeiros FA, Zangwill LM, Bowd C, Vasile C, Sample PA, Weinreb RN. Agreement between stereophotographic and confocal scanning laser ophthalmoscopy measurements of cup/disc ratio: effect on a predictive model for glaucoma development. J Glaucoma 2007; 16: 209-214. 